\title{
Cusp Prolapse Severity
}

National Cancer Institute

\section{Source}

National Cancer Institute. Cusp Prolapse Severity. NCI Thesaurus. Code C127554.

The qualitative measurement of the severity of a prolapsing cardiac valve cusp. 